\title{
BMJ Open Exploring the factors that promote or diminish a psychologically safe environment: a qualitative interview study with critical care staff
}

\author{
Kate Grailey (D) , ${ }^{1}$ Clare Leon-Villapalos (D) , ${ }^{2}$ Eleanor Murray (D) , ${ }^{3}$ \\ Stephen Brett (1D) ${ }^{1}$
}

To cite: Grailey K, LeonVillapalos C, Murray E, et al. Exploring the factors that promote or diminish a psychologically safe environment: a qualitative interview study with critical care staff. BMJ Open 2021;11:e046699. doi:10.1136/ bmjopen-2020-046699

- Prepublication history and additional supplemental material for this paper are available online. To view these files, please visit the journal online. (http://dx.doi.org/10.1136/ bmjopen-2020-046699)

Received 06 November 2020 Accepted 20 July 2021

Check for updates

(c) Author(s) (or their employer(s)) 2021. Re-use permitted under CC BY-NC. No commercial re-use. See rights and permissions. Published by BMJ.

${ }^{1}$ Department of Surgery and Cancer, Imperial College London, London, UK

${ }^{2}$ Department of Critical Care, Imperial College Healthcare NHS Trust, London, UK

${ }^{3}$ Said Business School, University of Oxford Said Business School, Oxford, UK

Correspondence to

Dr Kate Grailey;

k.grailey18@imperial.ac.uk

\section{ABSTRACT}

Objectives This study aimed to quantify the presence of psychological safety (defined as an environment 'safe for interpersonal risk taking') in critical care staff, exploring the ways in which this manifested.

Design Qualitative interview study incorporating a short quantitative survey.

Setting Three intensive care units within one National Health Service Trust in London.

Participants Thirty participants were recruited from all levels of seniority and roles within the multidisciplinary team. A purposive sampling technique was used, with recruitment ceasing at the point of thematic saturation. Interventions Semistructured interviews explored attitudes towards psychological safety and contained a quantitative assessment measuring the climate of psychological safety present.

Results Twenty-eight participants agreed that it was easy to ask for help, with 20 agreeing it is safe to take a risk on the team, demonstrating a strong perception of psychological safety in this group.

Our thematic analysis highlighted areas where the context influenced an individual's psychological safety including personality, culture and leadership. Possible negative consequences of psychological safety included distraction and fatigue for the team leader. We demonstrated that speaking up can be influenced by motivations other than patient safety, such as undermining or self-promotion. Conclusions Our data demonstrate reassuring levels of psychological safety within the participants studied. This allowed us to explore in depth the participant experience of working within a psychologically safe environment. We add to the current literature by uniquely demonstrating there can be negative consequences to a psychologically safe environment in the healthcare setting. We expand on the influence of context on psychological safety by developing a model, allowing leaders to identify which elements of context can be modified in order to promote speaking up. Team leaders can use these data to help foster a culture of openness, innovation and error prevention while minimising the risk of negative implications

\section{INTRODUCTION}

Psychological safety was originally defined in 1990 as an employee's 'sense of being able to
Strengths and limitations of this study

- We demonstrate that context is important in creating a psychologically safe environment and identify potential areas for change that organisations can influence in order to promote psychological safety.

- This study highlights that there can be unintended negative consequences of a psychologically safe environment within the healthcare environment.

- These data are useful for healthcare leaders wishing to create and promote an environment of psychological safety by identifying areas within their environment that are modifiable.

- The themes identified regarding context and the manifestation of a psychologically safe environment are likely to be generalisable across clinical departments.

- There is a risk of participation bias in this study; those who have an interest in safety or who have experienced high levels of stress may have been more willing to participate.

show and employ one's self without fear of negative consequences to self-image, status or career'. ${ }^{1}$ This concept has been built on by Edmonson $^{2}$ to define its application in work teams as 'an environment safe for interpersonal risk taking' and is reliably described as a positive influence on team working within organisations. An environment such as this influences and helps promote employee voice, commitment to the organisation and innovation within the workplace. ${ }^{34}$

The intensive care unit can be a busy, relentless and stressful working environment. Staff are required to care for the sickest population of patients within the hospital, often in rapidly changing clinical scenarios and emergencies. They also must contend with constant pressures to manage patient flow within the constraints of capacity.

Consequently, this is a good environment in which to study psychological safety, as it is 
a pressurised environment that offers the scope for it to be very psychologically safe or unsafe. In this setting, an environment with high levels of psychological safety helps to facilitate all team members being able to voice potential concerns regarding patient safety, as well as proposing ideas and innovations that might improve both patient care and the management of the clinical department.

High rates of medical error are an ongoing problem in the healthcare sector, ${ }^{5}$ with inadequate communication contributing to a significant proportion of cases. ${ }^{6}$ A cross-sectional survey study comparing error, stress and teamwork in medicine and aviation found that $50 \%$ of intensive care staff reported difficulty in discussing mistakes and that error was not handled well by managers at their hospital. ${ }^{7}$ A similar study ${ }^{8}$ found that physicians and nurses perceived their working environments differently, with nurses finding it more difficult to speak up than physicians. This study also investigated the impact of a strict hierarchy on the team members, finding that most of those studied felt it was easier to speak up if a flat hierarchy was in place. If an individual feels psychologically safe within their working environment, it should follow that errors and mistakes will be identified, raised and corrected earlier, thereby minimising the risk of significant harm to patients. A psychologically safe environment should also facilitate organisational learning when system failures are discussed openly within the team rather than being quietly corrected by an individual. ${ }^{9}$

This study used predominantly qualitative methodology to explore attitudes to safety and the presence of psychological safety in staff members working within the critical care environment. The subset of data we present in this paper pertains to psychological safety within this setting and how our findings may be applicable to other environments within the healthcare setting.

This study was designed to build on existing literature regarding the presence of psychological safety within the healthcare setting by exploring psychological safety at three levels within the working environment (individual, team and organisational). The influence of situational context on the perception of psychological safety was identified during an extensive literature review (completed within a body of work on psychological safety by $\mathrm{KG}$ as part of a $\mathrm{PhD}$ programme). During the analysis of papers included within this review, a pattern of situational context affecting psychological safety was identified, which has not been explored in detail within the current literature on psychological safety in healthcare. These contextual factors included having a setting conducive to speaking $\mathrm{up}^{10}{ }^{11}$ and the team having common goals for patient care. ${ }^{12}$ We aimed to further explore the idea of situational context and how each clinical scenario may have contextual factors that can impact psychological safety.

Psychological safety is typically regarded as a positive entity, with studies reporting it to be the most important contributing factor to good teamwork. ${ }^{13}$ The question has been raised within the literature: can the presence of psychological safety lead to negative consequences? ${ }^{9} \mathrm{An}$ environment of psychological safety (which by definition allows individuals to bring up problems and tough issues) may also facilitate behaviours that are detrimental to the organisation. A study exploring psychological safety and unethical behaviour in management undergraduates found cheating behaviour to be higher in teams with high levels of psychological safety, as this facilitated such ideas being raised. ${ }^{13}$ There are no similar studies in healthcare workers, with existing literature focusing on the benefits of psychological safety in the clinical environment. ${ }^{14}$ While we did not anticipate unethical behaviour to manifest in this participant group, we hope to contribute to our understanding of psychological safety in the healthcare setting by addressing a gap acknowledged within the existing literature, whether an environment that feels psychologically safe can have negative repercussions ${ }^{9} 1516$ and what format this might take in healthcare workers.

The objectives in this study were designed to first establish the presence of psychological safety within our participant group using a quantitative survey (nested within the qualitative interview) ${ }^{2}$ and subsequently apply a thematic analysis technique to our qualitative interview data to identify themes and concepts regarding the materialisation and impact of a psychologically safe environment.

These three objectives were as follows:

1. Establish whether our study participants believed themselves to be working in a clinical department that was psychologically safe.

2. Explore how context influences the presence of psychological safety within the clinical environment, and what contextual factors are important at different levels within the organisation?

3. Identify any potential consequences of working in a psychologically safe environment. In particular, do any perceptions of negative consequences or experiences of psychological safety exist?

\section{METHODS}

Members of the multidisciplinary team working within critical care (across all levels of seniority) were recruited according to a purposive sampling technique from three critical care units within one trust in London between January and June 2019. This sampling technique ensured those participating in the study reflected the multidisciplinary team working within the critical care department. ${ }^{17}$ Written informed consent was obtained from participants, with provision of written materials prior to the commencement of the qualitative interviews. The study protocol is available in online supplemental file 1.

\section{Data collection and analysis}

In line with qualitative methodology, interview data were continuously reviewed and analysed for similarities and differences using a constant comparative technique. ${ }^{18} 19$ This technique allowed themes to be identified and explored further as part of an iterative process. 
Participant recruitment ceased at the point of achieving thematic saturation, defined at the point at which no new codes were added to the evolving thematic framework. ${ }^{2021}$

Within the qualitative semistructured interview, participants were asked to complete a 'measure of climate for psychological safety' survey by stating their agreement with statements relating to psychological safety. ${ }^{2}$ The topic guide for these interviews was informed by a thorough literature search and refined by discussion within the research team (KG, EM and $\mathrm{SB}$ ). This (including the survey used to assess psychological safety) can be found in online supplemental file 2 .

Qualitative interview audio files were recorded and transcribed with all personal identifying information removed. These transcripts were analysed within qualitative software NVivo ${ }^{12}$ using a thematic analysis approach that followed the sequence of familiarisation with the data, subsequent construction of an initial thematic framework, indexing and sorting of the data, review of data extracts and finally data summary and display. ${ }^{22}$ Quantitative survey data assessing the presence of psychological safety were analysed using descriptive statistics within Microsoft Excel and GraphPad PRISM.

Ten per cent of the transcripts were coded by a second researcher (CL-V) and assessed for inter-rater reliability through calculation of the percentage agreement and the kappa coefficient (a statistical measure to determine the observed agreement between two raters ${ }^{23}$ ). This analysis was performed within NVivo. ${ }^{12}$ Discrepancies between coders were resolved through discussion within the wider research team.

\section{Reflexivity}

For reflexivity, KG is a clinical research fellow with a background in anaesthesia and critical care, CL-V is a senior nurse educator in critical care, EM is a former NHS manager who is now an academic in organisational studies and SB is a clinical academic and consultant in intensive care; all have previous experience with the conduct and analysis of qualitative studies in a clinical environment.

This manuscript is written in accordance with the Standards for Reporting Qualitative Research (checklist. ${ }^{24}$ This can be seen in online supplemental file 3 .

\section{Patient and public involvement}

No patients or public were involved in the design or implementation of this study.

\section{RESULTS}

Thirty participants across three critical care units were recruited; each completed Edmondson's safety survey and a semistructured qualitative interview. The interviews were all conducted in person and ranged between 00:21:49 and 01:05:12 in duration (with an average length of 00:38:39). The participant sample included 11 nurses, 3 physiotherapists and 16 doctors, encompassing all levels of seniority. Thematic saturation was defined by the point at which no new codes were added to the thematic framework, and no codes or themes were further modified or added as subsequent transcripts were analysed. This was done in line with existing literature regarding qualitative methodology. ${ }^{20}{ }^{21}$ Thematic saturation was achieved within the first 20 transcripts analysed, with only minor modifications made to the coding framework after this point.

Three $(10 \%)$ of the transcripts were selected at random to assess inter-rater reliability. A coding comparison query was performed with two outcomes: the kappa coefficient (how likely agreement is to be due to chance) and the percentage agreement. These data can be seen within online supplemental file 4 , with values for each of the theme and subthemes relating to psychological safety that were developed within the thematic framework constructed during the thematic analysis. The percentage agreement was high across all themes, with the kappa coefficient demonstrating excellent agreement $(\mathrm{K}>0.7)$ for eight of the themes and fair to good agreement $(\mathrm{K}=0.4-0.7)$ for three.

\section{The presence of psychological safety}

Our approach to exploring psychological safety within the participant group yielded a wealth of data. Within the seven statements ${ }^{2}$ used to evaluate whether they perceived a climate of psychological safety to be present, the majority of participants answered in a way that was consistent with their workplace being psychologically safe. In particular, 28 (93\%) felt confident that they could ask other members of the team for help, with the same number agreeing that no one in the team would deliberately undermine them. These results are displayed in figure 1 .

\section{The influence of context on the presence of psychological safety}

Once a climate of psychological safety had been identified in our participant sample by way of agreement with statements on Edmondson's psychological safety survey, qualitative interview transcripts were further examined to understand and develop themes regarding the influence of context on psychological safety. Some key subthemes emerged from this analysis, which were relevant at different levels within the workplace. These data can be viewed in full in online supplemental file 5 .

At an individual level, contextual factors that influenced psychological safety included location to speak up (this was particularly relevant if the setting was during a ward round with a large number of attendees), previous experience of speaking up and an individual's confidence in their ability.

Other contextual factors at an individual level were the impact of their personality (as perceived by the participant) and their culture (some participants felt that their ability to raise concerns was impeded by that fact they did not find it culturally acceptable to speak up against someone more senior). 


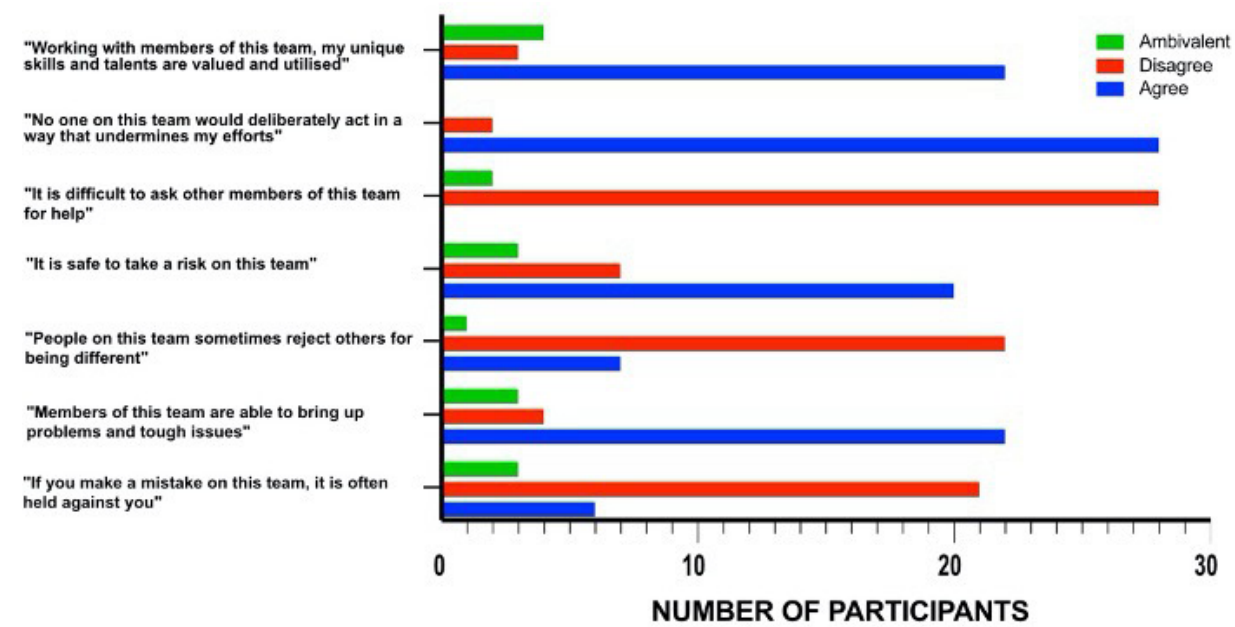

Figure 1 Participant agreement with statements pertaining to psychological safety.

At a team level, three areas of context emerged that affected an individual's psychological safety. This included the level of clarity regarding allocated roles within the team (this was a particularly predominant theme when discussing psychological safety within an emergency clinical scenario), presence of clear leadership and personality of other team members.

Context also had an influence at an organisational level, with factors influencing psychological safety that applied to all teams and individuals working within that environment being identified as prominent themes during the analysis. For example, environments with a stricter hierarchy were reported as creating a less psychologically safe space. The context of the culture within the organisation was also important (eg, if speaking up was encouraged within the organisation as a whole, as opposed to just within individual teams). This was reflected when looking at the context of organisational support. The provision of alternative routes to speak up as provided by the structure within the organisation also proved to be a prominent subtheme; using alternative routes was a method used by many individuals as a way to navigate around some perceived barriers to psychological safety.

A model demonstrating how these factors can influence the context of each clinical situation requiring psychological safety was developed and is shown in figure 2.

\section{Potential negative consequences of working in a psychologically} safe environment

One area explored within the qualitative interviews was a participant's experience of working in a psychologically safe environment. Within this, participants were asked directly if they had observed or experienced any negative consequences of psychological safety. While all participants were in agreement that the presence of psychological safety was important and generally beneficial within the critical care environment, there was an awareness from both those 'listening to concerns' and those 'taking risks' of the potential for negative consequences. This did not influence the feeling of being 'safe to take a risk on the team' itself as these consequences did not lead to the individual feeling unsafe or at risk of personal retribution as a result of taking a risk within the working environment. There were three key subthemes where negative consequences of psychological safety were described as arising: the impact on a clinical decision maker's 'bandwidth' (defined as the energy or mental capacity to deal with a situation), that the context of the clinical situation could lead to negative consequences when all individuals were psychologically safe and that a psychologically safe environment facilitated motivations to speak up that were not always regarded as beneficial to the organisation.

These subthemes regarding the possible negative consequences to psychological safety are outlined below, with supporting quotations for each subtheme illustrated in figure 3. Further supporting qualitative data can be viewed in online supplemental file 6 .

\section{Impact on 'bandwidth'}

It appeared consistent across all interviews that the person who was responsible for clinical decision making (this would vary depending on the clinical scenario, staffing

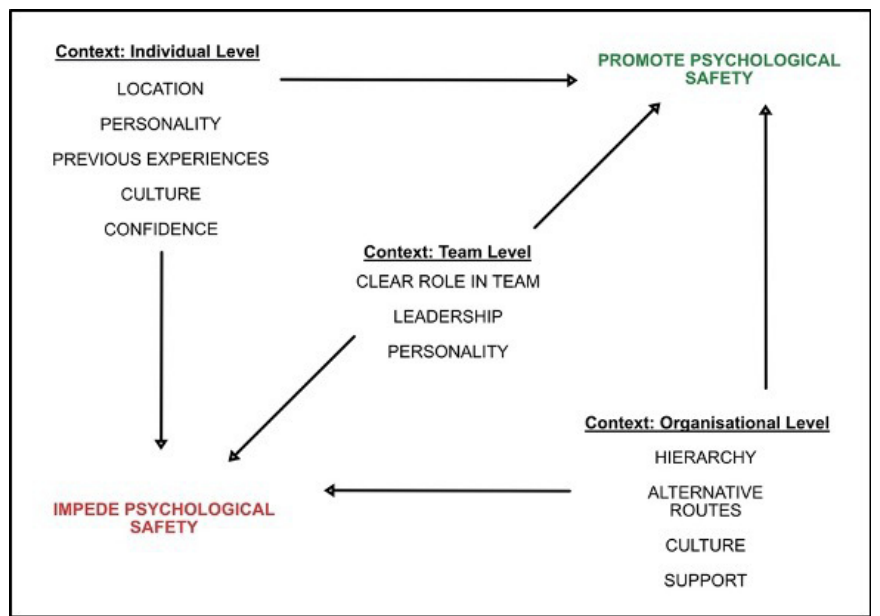

Figure 2 Contextual factors that can influence psychological safety and differ between clinical scenarios. 


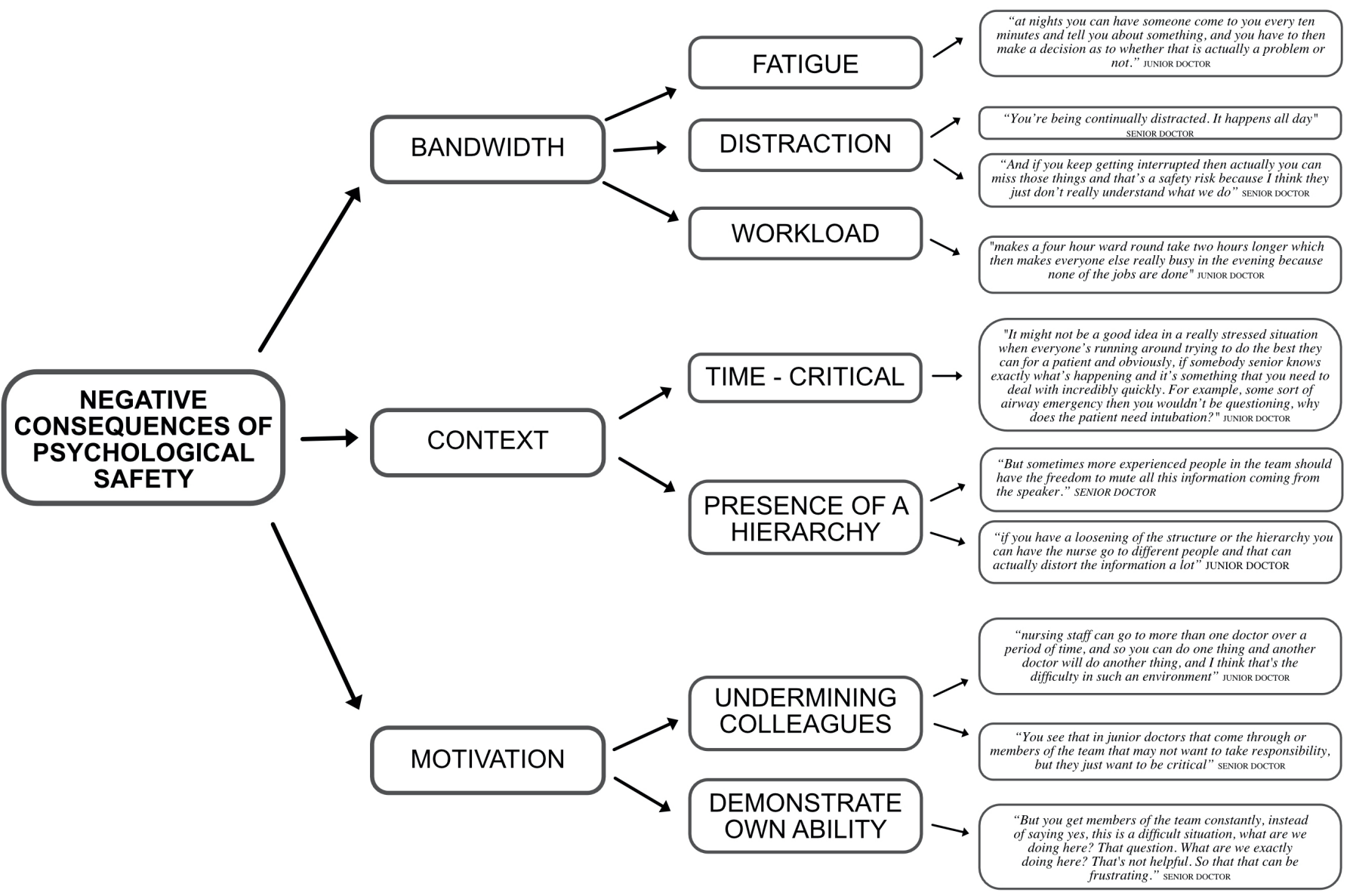

Figure 3 Themes that emerged from our analysis pertaining to the potential negative consequences of psychological safety.

and shift times) was at risk of having an overloaded 'bandwidth' as a result of multiple concerns or ideas being raised.

The impact on a clinical decision makers 'bandwidth' had three potential consequences. First, the decision maker became at risk of fatigue, potentially leading to poorer clinical decision making. Second, an increase in the volume of concerns raised causing the decision maker to become distracted, potentially missing important clinical information or making errors. Third, participants raised the issue that the clinical workload is often significant and by having to manage a large number of concerns being raised this could subsequently reduce the time available for clinical care (eg, by making the ward round longer in duration).

\section{Context}

Another subtheme was the context of the clinical situation. In an emergency situation, raising concerns that were not time-critical was felt to be negative and posed a risk of distracting the clinical decision maker from the current situation. Some individuals would voice concerns to multiple senior staff members, which had the potential to cause confusion and error.

Looking at the context of each clinical scenario, an environment perceived to have a flatter hierarchy was deemed to have an effect on facilitating some of the negative aspects of psychological safety. This more level structure allowed multiple voices and opinions to be heard, risking confusion and a distortion of the original clinical plan.

\section{Motivation to speak up}

The perception of being in a psychologically safe environment was described as providing individuals with the assurance and confidence to speak up and be an active member of the team, even if their motivations were not to promote good clinical care or improve the organisation.

Some participants observed that in others, this motivation may not have been driven by patient safety, but either to deliberately criticise or to undermine those in a more senior position. This also manifested as individuals raising issues to more than one person. In addition, individuals may use a psychologically safe environment in order to speak up and be 'seen' to be engaged and involved, knowing they can do this without fear of personal repercussion.

\section{DISCUSSION}

Broadly speaking, the participants in our study provided a positive account of their experiences and feelings of 
psychological safety within the critical care environment. The experiences reported and forming peoples' views will of course not just have been derived from their current position but will also encapsulate wider professional experience. Our data show that most participants were in agreement with statements that corresponded to high levels of psychological safety. By establishing that the participants included in this study perceived themselves to have a very good level of psychological safety, it allowed the exploration of how this manifests and what affects its presence. The strong presence of psychological safety also then allowed us to explore what the consequences of this environment might be, specifically whether any of these were negative.

Our first significant finding is the influence of situational context on psychological safety. These findings highlighted recurrent factors at the individual, team and organisational level that were specific to a healthcare setting. Understanding the role of context in every clinical scenario is important and an essential part of improving psychological safety within the clinical environment. Knowing what factors can influence the context ensures the clinical team possesses enough psychological safety to 'take a risk', thereby minimising the likelihood of clinical error and fostering a culture of innovation.

The model developed in this thematic analysis can assist team leaders in recognising the dynamic factors that influence the context of the situation and subsequently psychological safety within their own team. This information can be used to mitigate barriers and promote the factors that foster a culture of psychological safety.

While some factors affecting context may be fairly consistent, for example, the location and setting of the ward round and who leads it, others may vary from day to day depending on the individuals comprising the team. The nature of individuals' different personalities, cultural backgrounds and confidence influences the context. Team leaders can acknowledge this, providing encouragement and reassurance to their team when speaking up or proposing ideas. Workload also plays a role; clinical cases have varying complexity, and the volume of cases change from day to day. These factors interact with the clinical experience of members of the team and affect how psychologically safe they may feel.

The context of each clinical scenario can be affected by team dynamics. The impact of this on psychological safety can be improved by increasing the clarity of allocated roles and responsibilities.

Consistent factors (such as location to speak up) can also be identified and modified, recognising that a large ward round may inhibit some individual's psychological safety and consequently providing alternative routes and locations to speak up.

A second significant finding is the presence of potentially negative consequences of a psychologically safe environment. It is often assumed that psychological safety is universally beneficial and should be promoted in every situation. A recent review of psychological safety ${ }^{9}$ raised the question that there may be negative effects of psychological safety and have drawn attention to this as an area for future research. Our data provide evidence to corroborate this suggestion and highlight several ways in which high levels of psychological safety can have negative consequences, some of which are specific to the healthcare setting (eg, during emergency clinical scenarios) and others that are more generalisable to all workplaces (such as distraction of the team leader). These negative aspects only become prominent when a high level of psychological safety is present, as without an environment where individuals feel free to take a risk (eg, working within a psychologically safe culture that encourages speaking up to raise concerns), these negative aspects do not manifest.

These negative consequences manifested in three key subthemes within our analysis, which also allows potential solutions to be considered.

Many participants in senior positions found that they were at risk of distraction and fatigue, with their 'bandwidth' often becoming overloaded. This might be minimised by providing clear signposted opportunities for individuals to speak up, thereby reducing the risk of constant questioning and interruption.

There were two key areas within the subtheme of context that appeared to lead to negative consequences of psychological safety. In time-critical situations, perceived psychological safety allowed multiple opinions and ideas to be raised, taking up valuable time and risking patient safety. This may be improved by providing opportunities to contribute at a more appropriate time or by emphasising the time-critical nature of the situation.

Hierarchy also had an important influence. While a flatter hierarchy is known to promote psychological safety, conversely it may facilitate some of the negative aspects to psychological safety as demonstrated within our analysis. Many participants were in agreement that a steep hierarchy was a negative concept but also wanted clear leadership with the team. This clear leadership may be crucial in managing negative consequences of psychological safety, subsequently protecting the team leader.

The third subtheme demonstrated how a psychologically safe environment could facilitate voices and contributions with other motivations, such as undermining others and promoting oneself. It is difficult to truly ascertain an individual's personal motivation for speaking up, but it may be worthwhile for team leaders to bear this in mind if the psychologically safe environment is allowing a team member to speak freely, and this is taking the form of criticism, or that their negative opinions are becoming problematic for the functioning of the team as a whole.

Mitigating these negative consequences is not only the responsibility of the team leader, but of those within the team, using their own ability to weigh up the context of the situation, the urgency of the concern and the workload of the senior decision maker. It is worth remembering that these negative consequences must be managed and mitigated in a way that does not interfere with the psychological safety of the working environment, for example, 
the team leader clearly signposting opportunities for individuals to be an active member of the team but simultaneously minimising the risk of distraction and allowing space for decision making.

Our study has some limitations. While we achieved thematic saturation during the recruitment and analysis, it is still possible that themes regarding psychological safety within our recruitment sites were not represented. There is also the risk of participation bias; those with a proactive interest in safety and quality improvement (or conversely those who do not feel psychologically safe) may have been more willing to participate in this study.

There is scope to further explore the influence of context on psychological safety and the potential for negative consequences of a psychologically safe environment in clinical environments outside the critical care unit and within different NHS Trusts. While this study was performed within the critical care units of one large academically focused institution, the issues identified seem likely to be salient elsewhere and certainly worthy of wider exploration.

\section{Conclusions}

This study provides two unique contributions to the literature: through identifying and extending the role of situational context in psychological safety and second by introducing and expanding on the possible negative effects of a psychologically safe environment within the healthcare setting. By understanding the nuanced differences in facilitators and barriers within a particular workplace (eg, critical care within a wider model of healthcare) and how the context can change and also influence psychological safety, we may be able to develop environment specific and personalised solutions for departments to enhance the psychological safety of their workers.

While attainment of high levels of psychological safety within the team remains the goal, it is important to be aware there are negative consequences that arise as a function of being psychologically safe. By being aware of the possible negative aspects of general team psychological safety, team leaders and management can develop and institute systems in order to mitigate these while preserving an atmosphere in which people feel free to take risks and be active members of both the team and organisation as a whole.

\section{Twitter Clare Leon-Villapalos @cleonvillapalos}

Contributors KG, EM and SB conceptualised and designed this study. KG and SB were involved in recruitment of participants. $K G$ was responsible for data collection and overall data analysis. CL-V assisted with data analysis. All authors reviewed the data and coding discrepancies in line with qualitative research techniques. KG prepared the original draft manuscript. All authors reviewed and edited the manuscript. All four authors approved the final version of the manuscript. The corresponding author confirms that all listed authors meet authorship criteria and that no others meeting the criteria have been omitted. SB is the guarantor.

Funding KG received an unrestricted educational grant from BUPA Cromwell Hospital. Infrastructure support for this research was provided by the Imperial Comprehensive Biomedical Research Centre.

Disclaimer The funders had no role in study design, data collection and analysis, decision to publish or preparation of the manuscript.
Competing interests None declared.

Patient consent for publication Not required.

Ethics approval As per UK Research Governance guidance, studies involving National Health Service (NHS) staff by virtue of their role do not require ethical approval. Health Research Authority approval was granted for this study (Reference: 19/HRA/0522) in addition to approval from Imperial College Healthcare NHS Trust's and Imperial Colleges Joint Research Compliance Office (Reference: 18HH4812).

Provenance and peer review Not commissioned; externally peer reviewed.

Data availability statement All data relevant to the study are included in the article or uploaded as supplementary information. Deidentified participant data in the form of qualitative interview transcripts and completed quantitative survey data are stored in a secure server within Imperial College NHS Healthcare Trust. They are available from the author (ORCID ID 0000-0002-4104-8444).

Supplemental material This content has been supplied by the author(s). It has not been vetted by BMJ Publishing Group Limited (BMJ) and may not have been peer-reviewed. Any opinions or recommendations discussed are solely those of the author(s) and are not endorsed by BMJ. BMJ disclaims all liability and responsibility arising from any reliance placed on the content. Where the content includes any translated material, BMJ does not warrant the accuracy and reliability of the translations (including but not limited to local regulations, clinical guidelines, terminology, drug names and drug dosages), and is not responsible for any error and/or omissions arising from translation and adaptation or otherwise.

Open access This is an open access article distributed in accordance with the Creative Commons Attribution Non Commercial (CC BY-NC 4.0) license, which permits others to distribute, remix, adapt, build upon this work non-commercially, and license their derivative works on different terms, provided the original work is properly cited, appropriate credit is given, any changes made indicated, and the use is non-commercial. See: http://creativecommons.org/licenses/by-nc/4.0/.

\section{ORCID iDs}

Kate Grailey http://orcid.org/0000-0002-4104-8444

Clare Leon-Villapalos http://orcid.org/0000-0002-1610-0319

Eleanor Murray http://orcid.org/0000-0003-0939-6462

Stephen Brett http://orcid.org/0000-0003-4545-8413

\section{REFERENCES}

1 Kahn WA. Psychological conditions of personal engagement and disengagement at work. Academy of management journal 1990;33:692-724.

2 Edmondson A. Psychological safety and learning behavior in work teams. Adm Sci Q 1999;44:350-83.

3 Baer M, Frese M. Innovation is not enough: climates for initiative and psychological safety, process innovations, and firm performance. $J$ Organ Behav 2003;24:45-68 http://doi.wiley.com/10.1002/job.v24:1

4 UGGURLU ÖY, Sibel A. The relationship between psychological safety and employee voice: the mediation role of affective commitment and intrinsic motivation. Journal of Business Research-Türk 2016;8:223-39.

5 Elliott R, Camacho E, Campbell F. Prevalence and economic burden of medication errors in the NHS in England. In: Rapid evidence synthesis and economic analysis of the prevalence and burden of medication error in the UK, 2018.

6 AHA Hospital. Statistics 2018 Edition. Health Forum LLC, 2018

7 Sexton JB, Thomas EJ, Helmreich RL. Error, stress, and teamwork in medicine and aviation: cross sectional surveys. BMJ 2000;320:745-9.

8 Thomas EJ, Sexton JB, Helmreich RL. Discrepant attitudes about teamwork among critical care nurses and physicians. Crit Care Med 2003;31:956-9.

9 Frazier ML, Fainshmidt S, Klinger RL, et al. Psychological safety: a meta-analytic review and extension. Pers Psychol 2017;70:113-65 http://doi.wiley.com/10.1111/peps.2017.70.issue-1

10 Law BY-S, Chan EA. The experience of learning to speak up: a narrative inquiry on newly graduated registered nurses. J Clin Nurs 2015;24:1837-48.

11 Schwappach DLB, Gehring K. Silence that can be dangerous: a vignette study to assess healthcare professionals' likelihood of speaking up about safety concerns. PLoS One 2014;9:e104720.

12 Baik D, Zierler B. Clinical nurses' experiences and perceptions after the implementation of an interprofessional team intervention: a qualitative study. J Clin Nurs 2019;28:430-43. 
13 Rework with Google. Available: https://rework.withgoogle.com/print/ guides/5721312655835136/

14 Pearsall MJ, Ellis APJ. Thick as thieves: the effects of ethical orientation and psychological safety on unethical team behavior. $\mathrm{J}$ Appl Psychol 2011;96:401-11.

15 Bolino MC, Hsiung H-H, Harvey J, et al. "Well, I'm tired of tryin'!" Organizational citizenship behavior and citizenship fatigue. J Appl Psychol 2015;100:56-74.

16 Bolino MC, Turnley WH, Niehoff BP. The other side of the story: reexamining prevailing assumptions about organizational citizenship behavior. Human Resource Management Review 2004;14:229-46.

17 Robinson RS. Purposive sampling. In: Michalos A, ed. Encyclopedia of quality of life and well-being research. Dordrecht Netherlands: Springer, 2014: 5243-5.

18 Fram SM. The constant comparative analysis method outside of grounded theory. Qualitative Report 2013;18:1.
19 Harding J. Making comparisons of interview data and drawing out themes: experiences of working in higher education. SAGE, 2015.

20 Lowe A, Norris AC, Farris AJ, et al. Quantifying thematic saturation in qualitative data analysis. Field methods 2018;30:191-207.

21 Ando $\mathrm{H}$, Cousins R, Young C. Achieving saturation in thematic analysis: development and refinement of a codebook. Comprehensive Psychology 2014;3:03.CP.3.4-4.

22 Braun V, Clarke V. Using thematic analysis in psychology. Qual Res Psychol 2006;3:77-101.

23 Landis JR, Koch GG. The measurement of observer agreement for categorical data. Biometrics 1977;33:159-74.

24 O'Brien BC, Harris IB, Beckman TJ, et al. Standards for reporting qualitative research: a synthesis of recommendations. Acad Med 2014;89:1245-51. 